\title{
Timing and Frequency Synchronisation for Multiuser Detection on the Return Link of Interactive Mobile Satellite Networks
}

\author{
Zohair Abu-Shaban *, Bhavani Shankar M. R.*, Dimitrios Christopoulos* \\ and Pantelis-Daniel Arapoglou ${ }^{\dagger}$ \\ * SnT - securityandtrust.lu, University of Luxembourg \\ ${ }^{\dagger}$ ESA/ESTEC
}

\begin{abstract}
Synchronization, a key process in a communication system, can be very demanding, especially in burst mode transmissions. The estimation of timing, frequency and phase offsets is sensitive to the magnitude of these offsets as well as the ambient interference and noise levels. The aggressive reuse of frequencies in a multibeam satellite system increases the co-channel interference significantly and motivates the need to revisit, and re-design if needed, the algorithms used for synchronization. This paper deals with such a scenario occurring on the return link of a mobile interactive satellite system and provides a set of synchronization sub-systems that allow for the design of a robust modem that can reap the benefits of sophisticated interference mitigation techniques.
\end{abstract}

\section{Introduction}

With the proliferation of the mobile paradigm, there is an increased demand for providing multimedia applications and personalized services to the user on the move. Interactive services tend to contribute significantly to this demand. Such a development impacts satellite services positively by opening up new opportunities in addition to the traditional broadcasting and has motivated efforts towards standardization of mobile interactive satellite networks $[1,2]$. However, the increase in demand is not complemented by a proportional increase in the availability of spectrum warranting the need to explore architectures and techniques that make the system spectrally efficient. Towards this, multibeam architecture in conjunction with sophisticated signal processing on-ground have been considered. In systems that employ multiple beams, capacity increase is limited by the signals originating from different users in the same or adjacent spot beams. Such a scenario can occur due to co-channel interference (CCI) caused by inter-beam interference or adjacent channel interference (ACI) caused by aggressive carrier spacing.

Focussing on the return link (RL) of a mobile satellite system [2], in order to fully exploit the available spectrum, different multiuser detection (MUD) techniques can be applied at the gateway to mitigate the interference and detect the users data more efficiently with a tolerated bit error rate. Towards improving the spectral efficiency by reducing the carrier spacing between two RL time division multiple access (TDMA) carriers, iterative interference cancellation techniques have been considered in [3]. On a similar note, the authors in [4] increase the number of carriers per transponder and employ a MUD at the gateway (processing all the carriers jointly) to reduce the ACI caused by increased carrier overlap. On the other hand, Minimummean-squared error with successive interference cancellation (MMSE-SIC) is applied against CCI is caused by frequency reuse in [5].

A key challenge in MUD-based techniques is the timing, phase and frequency synchronisation. Timing error is caused by uncertainty associated with the optimal sampling instance. On the other hand, the transmitter and the receiver oscillators are not guaranteed to produce exactly the carrier frequency for up-conversion and down-conversion, respectively. This, coupled with the uncertainty associated with the oscillator phase and compounded by the relative mobility between the user terminals and a satellite, imposes phase and frequency offsets and can deteriorate the system performance. In order to improve the performance

*Email: zohair.abushaban@uni.lu, 4 rue Alphonse Weicker, L-2721 Luxembourg Tel +352 4666445897 
in presence of imperfections, efficient synchronization systems need to be incorporated. The synchronization challenge is accentuated by the burst mode transmissions on the RL which require rapid estimation and compensation of these offsets. The trend in literature has been to focus either on the synchronization aspects assuming absence of ACI/ CCI or on the derivation and application of MUD techniques assuming perfect synchronization. For example, [2,4] assume perfect synchronization while a large body of works [6-8] provide techniques for acquisition in packet oriented systems. The synchronization techniques consider variations of the well-known O\&M algorithm [9] as well as those obtained from the derivation of Cramer-Rao bounds. To the best of the authors' knowledge, only [3] discusses the incorporation of synchronization sub-systems along with MUD on the RL.

In this paper we devise timing, phase and frequency synchronisation for joint processing of carriers from co-channel beams. This work can be considered as an extension of [5] due to its inclusion of practical imperfections; it further complements [3] by dealing with CCI. To enhance the novelty, we consider the Broadband Global Access Network (BGAN) system model of [4] which is currently being standardized [2]. This set-up employs burst transmission in a multibeam system and different frequency reuse factors are considered towards generating CCI. Central to the synchronisation and subsequent processing is the pilot pattern (also known as Unique Word in [2]). We show that the synchronization is poor when employing the current pattern due to its inability to discriminate users. A simple modification based on scrambling is proposed to improve the acquisition performance. A hybrid timing synchronization algorithm where the estimates from non-data aided (NDA) Oerder\&Meyr algorithm [9] is further refined by the use of a data aided binning is also proposed. Frequency and phase offset estimators are derived using the Maximum Likelihood (ML) estimation and the performance of various estimators are then evaluated.

\section{System Description}

A BGAN-like narrowband mobile satellite system for interactive services depicted in figure 1 is considered. The multiple user terminals (UT) operate in the $L$ band $(1626.5 \mathrm{MHz}-1660.5 \mathrm{MHz}$ and $1668.0 \mathrm{MHz}-$ $1675.0 \mathrm{MHz}$ ) supporting continuous and burst mode transmissions through dedicated bearers (or carriers). These carriers are capable of carrying nominal data rates in the range between 3.2 to $848 \mathrm{~kb} / \mathrm{s}$ with the maximum channel bandwidth being $200 \mathrm{kHz}$. In this paper we assume that the power amplifiers at the UT and gateway $(\mathrm{GW})$ are operated in a linear mode so as not to cause additional interference.

\section{II.A. Space Segment and its Modelling}

A GEO satellite with 210 beams is considered with frequency reuse factors of 2 and 4 . For the purpose of this paper, we consider only the first tier of co-channel beams, depicted in figure 2 for the two reuse factors. To model the multibeam antenna radiation pattern in such a scenario, an approximation using Bessel functions [10] is employed wherein the gain coefficients in each beam are calculated as $g_{i j}\left(\theta_{i j}\right)=$ $\sqrt{G_{\max }\left(\frac{J_{1}(u)}{2 u}+\frac{J_{3}(u)}{u^{3}}\right)}$ with $u=2.07123 \sin \left(\frac{\theta}{\theta_{3 d B}}\right)$, and $J_{1}, J_{3}$ are respectively, the first and third order Bessel functions of the first kind. The $j^{\text {th }}$ user corresponds to an off-axis angle $\theta_{i j}$, with respect to the bore sight of the $i^{\text {th }}$ beam; clearly $\theta_{i i}=0^{\circ}$. Subsequently, beam gain for each satellite antenna - user pair, depends on the spot beam antenna pattern and on the user position. For ease of analysis, we assume that user position does not change considerably over the duration of a codeword. Thus, the position dependant beam gain between the $K$ users and the $N_{t}$ antennas can be represented by a $N_{t} \times K$ deterministic real positive matrix, $\mathbf{B}$, where the $(i, j)$ element of $\mathbf{B}$ denotes the gain accorded by the beam $i$ to an UT in beam

$j$. Hence, we have, $\mathbf{B}(i, j)=\sqrt{\frac{g_{i j}\left(\theta_{i j}\right)}{G_{\max }}}$. The $\mathrm{C} / \mathrm{I}$ values for the reuse 2 and 4 at the centre of the beam are provided in Tables 1 and 2 respectively.

Table 1. C/I values at the Centre of Beam for Reuse 2

\begin{tabular}{|c|c|c|c|c|c|c|c|c|c|c|c|}
\hline Beam & 1 & 6 & 7 & 5 & 9 & 10 & 11 & 8 & 4 & 3 & 2 \\
\hline C/I (dB) & 3.4 & 3.4 & 3.4 & 6.4 & 6.2 & 3.5 & 6.2 & 6.4 & 6.2 & 3.5 & 6.2 \\
\hline
\end{tabular}




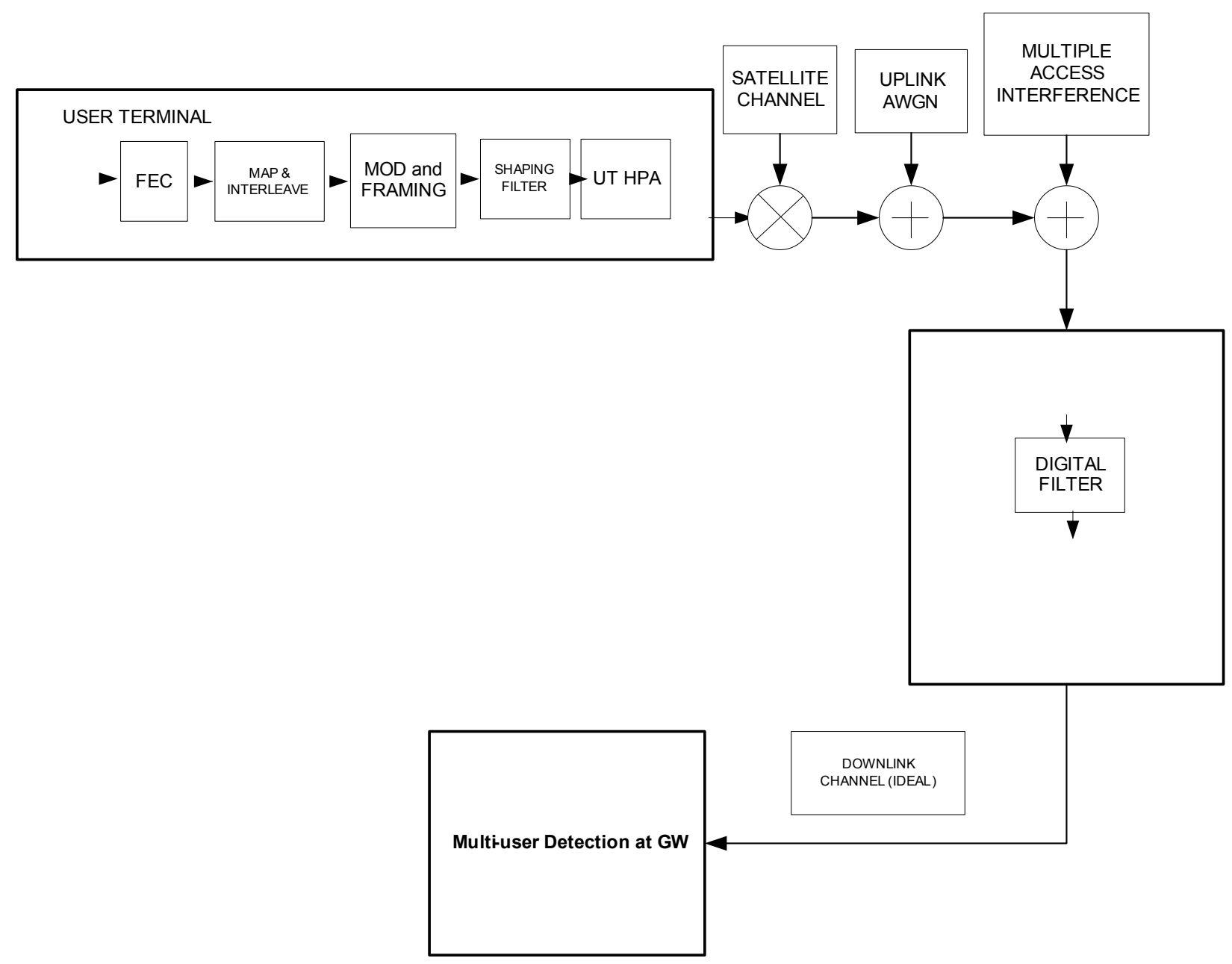

Figure 1. Generic system model used in the activity

Table 2. C/I values at the Centre of Beam for Reuse 4

\begin{tabular}{|c|c|c|c|c|c|c|c|}
\hline Beam & 1 & 2 & 3 & 4 & 5 & 6 & 7 \\
\hline C/I $(\mathrm{dB})$ & 19.5 & 18.8 & 18.9 & 18.9 & 18.8 & 18.7 & 18.7 \\
\hline
\end{tabular}

\section{II.B. Ground Segment and its Modelling}

The ground segment consists of the transmitting UTs and the receiving GW. Since the focus of this work is on synchronization, only the relevant functionalities are described.

\section{II.B.1. User Terminal}

While UTs can be classified based on the RF characteristics, we focus on the intermediate gain maritime UT having a minimum EIRP of $15 \mathrm{dBW}$. The diagram of the generic UT transmitter with relevant blocks is shown in figure 3 .

Modulator Two types of modulations namely $\frac{\pi}{4}$ QPSK and 16 QAM are considered with the constellation points and the corresponding mapping obtained from [2].

Burst fORmats A key feature of the RL traffic is its bursty nature [2]. Burst formats with 5, 20 and 80 ms slots are described in [2] and we consider the $20 \mathrm{~ms}$ slots since it serves as the principal data carrying burst. The structure of the burst is shown in figure 4 and consists of a guard interval (GI) followed by a preamble (denoted as CW in the figure). The CW field is used to provide a smooth ramping up of power to avoid violating ACI specifications by the sudden appearance of the carrier power at the input of the amplifier 


\begin{tabular}{|l|l|l|l|l|}
\hline & & & & \\
\hline & 5 & & 6 & \\
\hline & & & & \\
\hline 4 & & 1 & & 7 \\
\hline & & & & \\
\hline & 3 & & 2 & \\
\hline & & & & \\
\hline
\end{tabular}

(a)

\begin{tabular}{|l|l|l|l|l|}
\hline & & & & \\
\hline & 9 & 10 & 11 & \\
\hline & & & & \\
\hline 5 & 6 & 1 & 7 & 8 \\
\hline & & & & \\
\hline & 2 & 3 & 4 & \\
\hline & & & & \\
\hline
\end{tabular}

(b)

Figure 2. First tier CCI beams for different frequency reuse factors : (a) reuse 2, (b) reuse 4 . In red the center beam where the intended UT is located and in orange the interfering beams.

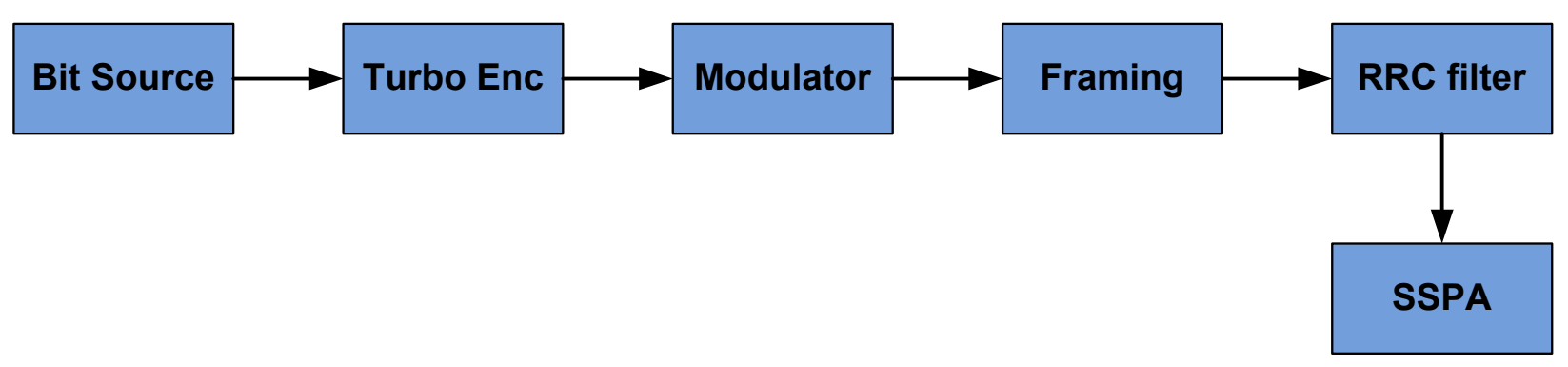

Figure 3. Block Diagram of the UT Transmitter

and is not prescribed for synchronisation [2]. Unique Words (UW), which follow the CW field, are used to signal the different FEC blocks used; further, they are used for frequency, phase and clock synchronization. Typically, each UW consists of 64 bits (rotated BPSK) for $\frac{\pi}{4}$ QPSK and 60 bits for 16 QAM; further, the code-book size of the UWs is 15 [2]. The UWs are split into start and end sections with the start section having 40 bits. UW bits are mapped onto symbols depending on the modulation used in the bearer.

$20 \mathrm{~ms}$

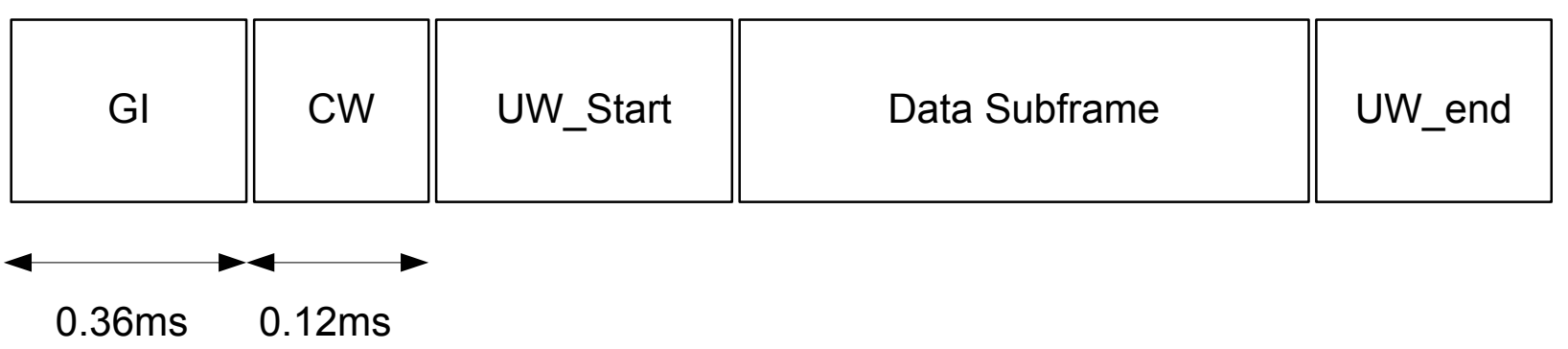

Figure 4. Frame format of the $20 \mathrm{~ms}$ Return Burst

Pulse shaping, Amplification and Upconversion A roll-off factor of 0.25 is assumed for the Root Raised Cosine pulse shaping filter and the amplifier is assumed to operate in the linear region. Further, the up-conversion assumes a frequency offset of $\triangle f_{i}$ for the $i^{\text {th }} \mathrm{UT}$.

\section{II.B.2. $\quad G W$ Processing}

The generic receiver structure used depicted in figure 5 , where $T s$ refers to the sampling rate. The relevant blocks comprise synchronization subsystems followed by joint processing of co-channel signals (MUD) where 
the aspects of FEC decoding are also incorporated. The synchronisation sub-systems are detailed in the sequel.

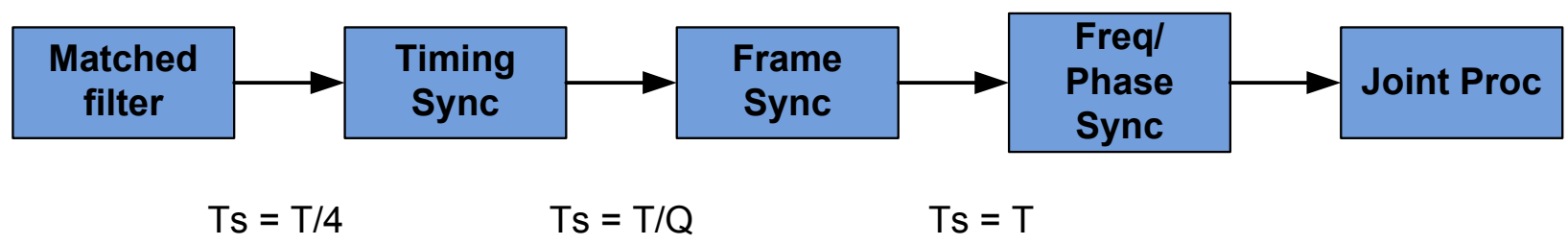

Figure 5. Detailed GW receiver architecture

\section{II.C. Channel}

Each beam is assumed to be served by a dedicated onboard feed/ antenna and the user uplink channel is modelled as a Rician fading channel. The signals from different co-channel antennas/feeds are multiplexed on the feeder downlink which is further assumed to be ideal. We also assume that only one user is served per beam at a particular time instance. The resulting set-up can be abstracted as a $N \times N$ multiple-input multiple-output (MIMO) system where $N$ is the number of co-channel users and the equivalent channel at time instance $t$ takes the form,

$$
\mathbf{H}(t)=\gamma \mathbf{B}(t) \mathbf{G}(t),
$$

where $\mathbf{G}(t)$ is a diagonal matrix comprising the uplink channel parameters at instance $t$ and $\gamma$ is the power normalization factor. Note that we have used the time dependence of $\mathbf{B}$ to reflect the mobility. Let $\underline{s}_{k}(t)$ be the symbol transmitted from user $k$ at time $t$ and $\underline{s}(t)=\left[\underline{s}_{1}(t), \underline{s}_{2}(t), \ldots, \underline{s}_{N}(t)\right]^{T}$ be the $N \times 1$ vector obtained by stacking the signals transmitted from $N$ co-channel users. Similarly, defining $y(t)$ to be the corresponding received vector and letting $\triangle f_{k}, \omega_{k}$ to be the frequency and phase offsets, respectively, for user $k$, the input-output relation then takes the form,

$$
\begin{aligned}
\underline{y}(t) & =\boldsymbol{\Lambda}(t) \mathbf{H}(t) \underline{s}(t-\underline{\tau} T)+\underline{\eta}(t), \\
\boldsymbol{\Lambda}(t) & =\operatorname{diag}\left(\left\{e^{-j 2 \pi\left(\triangle f_{k}\right) t+\omega_{k}}\right\}_{k=1}^{N}\right),
\end{aligned}
$$

where $\eta(t)$ is the receiver thermal Gaussian noise, $\boldsymbol{\Lambda}(t)$ is the diagonal matrix encompassing frequency and phase offsets and $\underline{s}(t-\underline{\tau} T)=\left[\underline{s}_{1}\left(t-\tau_{1} T\right), \underline{s}_{2}\left(t-\tau_{2} T\right), \ldots, \underline{s}_{N}\left(t-\tau_{N} T\right)\right]^{T}$ reflects the time offset. Here, $T$ $T$ is the symbol duration and $\left\{\tau_{l}\right\}$ are the normalized offsets. Note that the model supports different offset parameters for different users.

\section{Synchronization Subsystems}

In this section we describe the various synchronization algorithms employed. Both feedforward and iterative mechanisms in conjunction with MUD can be considered. While iterative techniques can improve the performance, such an implementation is complicated by the absence of a priori knowledge of the UW and also the use of complex MUD. Hence feedforward mechanism is considered and the offsets are estimated and compensated for each user stream separately. The standard methodology of correcting the timing offsets first, and then effecting by frequency and phase offset compensations is followed. However, some subtle changes are incorporated in the techniques per-se and the same are described below.

\section{III.A. Timing Synchronization}

The Oerder and Meyr (O\&M) algorithm [9] has been considered for synchronizing burst mode transmissions in $[3,6,7]$. The timing offset estimate for the $k^{t h}$ user takes the form,

$$
\widehat{\tau_{k}}=-\frac{1}{2 \pi} \angle\left\{\sum_{l=0}^{M_{0} P}\left|\underline{y}_{k}\left(\frac{l T}{M_{0}}\right)\right|^{2} e^{\frac{-j 2 \pi l}{M_{0}}}\right\},
$$

where $M_{0}$ is the oversampling factor (typically $M_{0}=4$ ) and $P$ is the integration window in symbol periods. However, it has been observed in [3] that the performance of O\&M algorithm is degraded in presence of ACI. 
Further, a technique exploiting the preamble structure, known as dichotomic search with linear interpolation has been suggested as an alternative [3]. Also, it is also shown in [6] that the unbiased region of the O\&M algorithm is limited and needs to be enhanced using data aided techniques. These motivate the following hybrid strategy pursued in the paper

- Without loss of generality, we assume $\tau_{k} \in[-0.5,0.5]$

- O\&M Estimate: Obtain O\&M timing estimates $\widehat{\tau_{k}}$ using equation (4)

- Binning: Quantize $\widehat{\tau_{k}}$ into one of the $2^{Q}$ levels in the range $[-0.5,0.5]$ where $Q \geq 4$. Define the resulting estimate as $\widetilde{\tau_{k}}$

- Effecting Timing Correction: Upsample and interpolate $\underline{y}_{k}\left(\frac{l T}{M_{0}}\right)$ to a rate $\frac{T}{Q}$, effect the timing correction of $\widetilde{\tau_{k}}$. Define the resulting signal as $\underline{x}_{k}(l)=\underline{y}_{k}\left(\frac{l T}{Q}+\widetilde{\tau_{k}}\right)$

Once the stream $\underline{x}_{k}(l)$ is obtained, a frame synchronization is undertaken (to be described in Section III.B). The proposed algorithm has similarities to the dichotomic sampling of [3] and its performance depends on the choice of $Q$. The choice of $Q$ results in a complexity-performance trade-off. While the steps leading to $\widehat{\tau_{k}}$ do not exploit CCI, the quantization step allows for a further refinement through frame synchronization where the UW correlation is exploited to mitigate CCI.

\section{III.B. Frame Synchronization}

The frame synchronization is carried out in the upsampled domain essentially by correlating with the UW. To keep the presentation simple, we assume that the start and end parts of the UW are concatenated into a single field. The proposed methodology can be used with straightforward variations to the frame structure depicted in figure 4. Frequency offset can degrade the performance of a mere correlation based technique. Motivated by post detection integration techniques in [11], the frame synchronization algorithm takes the form,

- Upsample the $U W$ : Let $u_{m}(n)$ denote the $n^{\text {th }}$ symbol of $\underline{u}_{m}$ and $U W_{L e n}$ is the length of the UW. Further, let $\underline{u}_{m}$ denote a stacking of the symbols corresponding to $m^{\text {th }}$ UW. Define the upsampled UW as $\underline{\underline{u}}_{m}=\underline{u}_{m} \otimes \underline{1}$ where $\underline{1}$ is $1 \times Q$ vector of ones and $\otimes$ denotes the Kronecker product.

- One tap UW correlation: Obtain $\underline{t}_{m}(n)=\widehat{\underline{u}}_{m}(n)\left[\underline{\widehat{u}}_{m}(n+1)\right]^{*}$ where ${ }^{*}$ denotes complex conjugation. From the form of UWs (rotated BPSK [2]), it can be easily verified that $\underline{t}_{m}(n)= \pm \alpha, \alpha>0$.

- One tap received signal correlation: Obtain $\underline{r}_{m}(n)=\underline{x}_{m}(n)\left[\underline{x}_{m}(n+1)\right]^{*}$.

- Fourth order correlation: Obtain $\beta_{m, k}(p)=\sum_{n=1}^{U W_{L e n}-1} \underline{t}_{m}(n)\left[\underline{r}_{k}(n+p)\right]^{*}$.

- Frame Synchronisation: The frame offset for $m^{t h}$ is obtained as $n_{m}=\arg \max _{p}\left(\max _{m}\left|\beta_{m, k}(p)\right|\right)$. Note that we search for the peak of the correlation over all the UWs and this step reflects the lack of a priori information about UW used. The motivation for this technique stems from the fact that, $\underline{r}_{m}(n)=e^{j 2 \pi[\Delta f]_{m}} \widetilde{\underline{\underline{r}}}_{m}(n)$ where $\underline{\underline{\widetilde{r}}}_{m}(n)$ is independent of the frequency offset when noise and CCI terms are neglected and $\left|\beta_{m, k}(p)\right|$ will be independent of the frequency offset.

- Instead of peak picking, a detection mechanism is put in place where we look at the peak only if

$$
\max _{p}\left(\max _{m}\left|\beta_{m, k}(p)\right|\right)>T H R
$$

for some threshold THR. If equation (5) fails then we consider it to be a failure. On the other hand if equation (5) holds and the detector provides wrong values for the beginning of the frame, we declare a false alarm. These events would be useful when plotting the Receiver Operating Characteristics.

Once the frame is synchronized, the signal is resampled to the symbol duration and we denote the symbol sampled stream as $\underline{\underline{x}}_{m}$. 


\section{III.C. Joint Frequency and Phase Synchronization}

For obtaining the estimates of frequency and phase offsets, we assume the CCI as an additional source of Gaussian noise that is independent of the signal and the receiver noise. Under such an assumption, it is possible to derive the joint Maximum Likelihood (ML) estimate of frequency and carrier offsets as solutions to

$$
\operatorname{Imag}\left\{\sum_{l=1}^{U W_{L e n}} e^{-j 2 \pi\left[\Delta f_{m}\right](l-1)}\left[\underline{\widehat{\widehat{x}}}_{m}(n)\right]^{*} \underline{u}_{m}(n)\right\}=0 .
$$

Equation (6) involves root finding which could be cumbersome. Motivated by $|\operatorname{Imag}(y)| \leq|y|$ and resorting to finding the minimum instead of the roots, we use the following methodology to obtain the estimates,

$$
\begin{aligned}
\triangle \widetilde{f}_{m} & =\frac{1}{\operatorname{Len}} \arg \min _{k}\left|G_{\text {Len }}^{m}(k)\right|, \\
\left\{G_{\text {Len }}^{m}(k)\right\}_{k} & =F F T\left(\left[\widehat{\widehat{x}}_{m}(n)\right]^{*} \underline{u}_{m}(n), \quad \text { Len }\right), \\
\widehat{\omega_{m}} & \left.=\angle\left\{\sum_{l=1}^{U W_{\text {Len }}} e^{-j 2 \pi\left(\left[\Delta \widetilde{f}_{m}\right](l-1)\right)} \underline{\widehat{\widehat{x}}}_{m}(n)\right]^{*} \underline{u}_{m}(n)\right\} .
\end{aligned}
$$

In equation (8), $\left\{G_{\mathrm{Len}}^{m}(k)\right\}_{k}$ refers to the Len length Fast Fourier Transform (FFT) of the sequence $\left\{\left[\underline{\underline{\widehat{x}}}_{m}(n)\right]^{*}\right.$ $\left.\underline{u}_{m}(n)\right\}$.

\section{Simulation Results}

We evaluate the performance of the proposed estimators by simulating the proposed chain with the uplink modelled as a Rician channel with $K=15 \mathrm{~dB}$ and a fading bandwidth of $2 \mathrm{~Hz}$ to emulate a maritime channel. Focusing on users located at the centre of the beam, we present the results for the user in the central beam (worst case CCI). In all the simulations, we assume a single slot per burst. It should be recalled that different users can have different UWs and these need to be estimated the GW as well. The frequency offset is chosen as $\triangle f_{i}=\frac{0.015}{T}, \forall i$ and the phase offset is chosen uniformly in $[0,2 \pi]$. Further, we assume the phase offset to be constant during the duration of the UW.

\section{IV.A. Frame Synchronization}

We assume perfect timing and illustrate the probability of detecting the start of frame correctly for different SNRs in presence of frequency offsets. The aim of this exercise is to illustrate that the selection of UWs has a significant effect on frame, phase and frequency synchronization. The probability of detection is obtained by averaging over $10^{5}$ bursts. To put the SNR range in perspective, it should be noted that the supported codes achieve a packet error rate of $10^{-3}$ for a SNR range of [3-15]dB when employing 16-QAM constellation [2]. From figure 6, it can be seen that the frame detection with the current set of UWs is rather poor (legend : Existing UW) and requires additional processing to improve performance. On the other hand, a simple modification where the UWs are scrambled using \pm 1 yields a much better performance (legend : Modified UW). Further, the performance of reuse 2 is poorer than reuse 4 as expected. Also depicted in figures 7 and 8 are the Mean Squared Error(MSE) of the normalized frequency and phase offsets respectively.

These results indicate that the existing UWs do not discriminate between users well. Henceforth, we consider using the modified UW. It should be noted that the scrambling sequence has been chosen ad-hoc and there exists scope for further optimization.

\section{IV.B. Timing Synchronization}

Figure 9 illustrates the performance of the proposed timing algorithm for $\tau_{1}= \pm \frac{k}{16 T}, k \in[0,7], \tau_{l}=0, l>$ $1, Q=8$ at $E_{s} / N_{0}=0 \mathrm{~dB}$. The mean of the estimated normalized offset closely follows the true value over the considered range. The apparent bias in the estimation arises from the quantization and is generally of the order $1 / Q$. This is implicitly removed when the UW aided frame synchronization is performed. It should be noted that the resulting frame synchronization is achieved with an average probability of $99.99 \%$ over the chosen range of $\tau_{1}$. Further, the MSE of the frequency and phase offsets are $10^{-4}$ and $10^{-2}$ respectively 


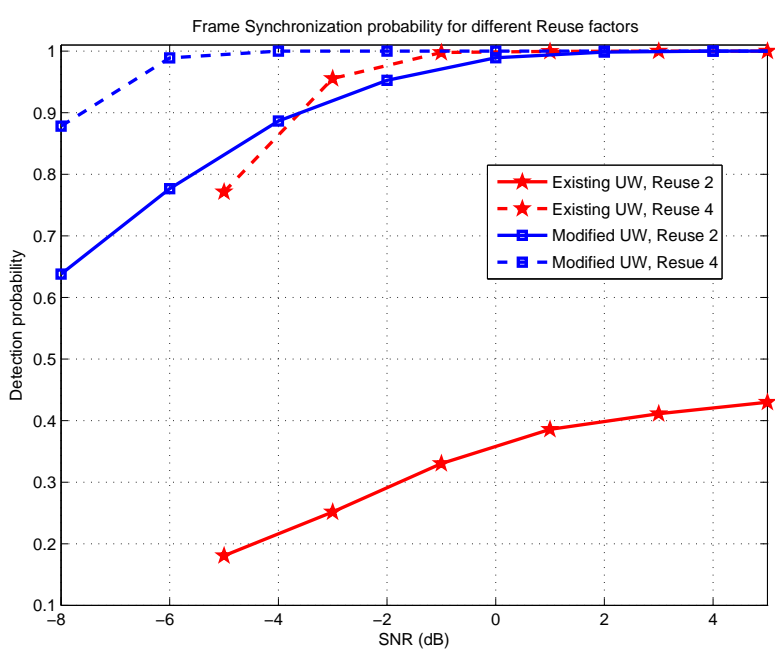

Figure 6. Frame Detection probability at different SNR, normalized frequency offset of 0.015

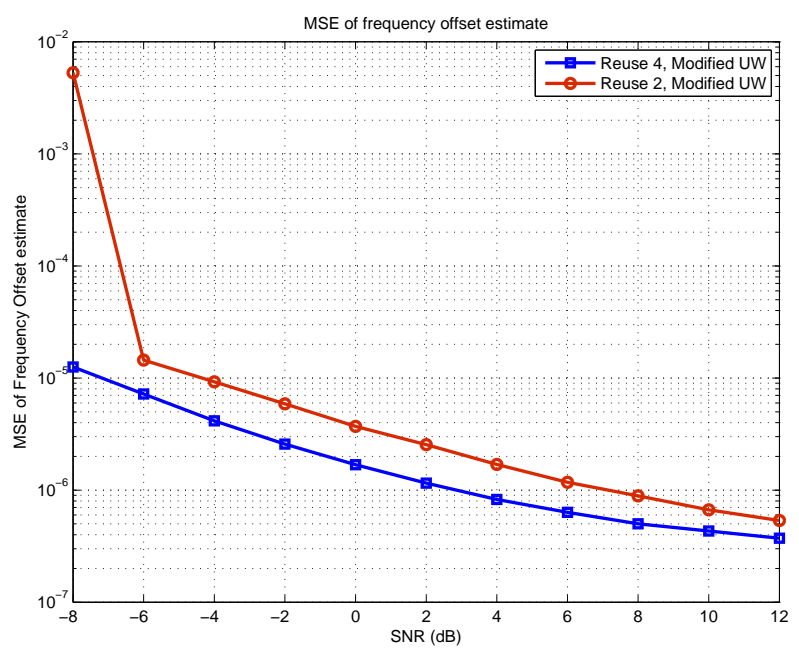

Figure 7. MSE of the normalized frequency offset estimate at different SNR, normalized frequency offset of 0.015

over the considered range. These results indicate the effectiveness of the various synchronization algorithms in overcoming the imperfections.

\section{Conclusions}

This paper deals with the design of synchronisation algorithms for the return link of a mobile interactive satellite network. A hybrid timing synchronization algorithm where the non-data aided estimate is refined by exploiting the pilot pattern is proposed along with a start of frame detector that is robust to frequency offsets. Further, a FFT based frequency estimator and a low complexity phase estimator are also provided and their performance is evaluated. Key to the performance of these algorithms is the ability to correctly detect the start of frame which further requires a better design of pilot pattern (unique words) than the currently prescribed. 


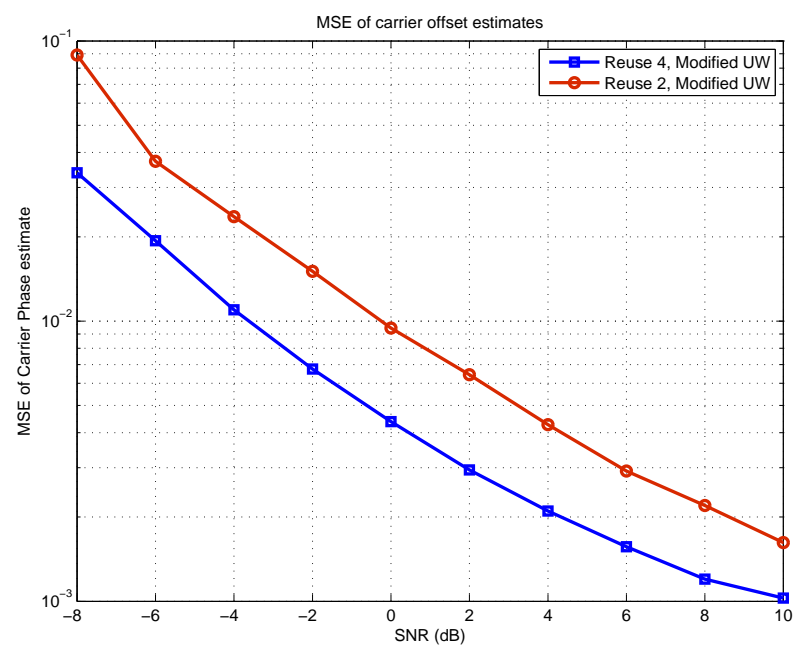

Figure 8. MSE of the phase offset estimate at different SNR, normalized frequency offset of 0.015

\section{Acknowledgment}

Part of this work is supported by the ESA Contract. No. 40001 06528/12/NL/NR, "Next Generation Waveforms for Improved Spectral Efficiencies". Zohair Abu-Shaban is supported by the National Research Fund (FNR), Luxembourg under AFR scheme. Project ID: 4043055.

\section{References}

${ }^{1}$ DVB, "Interaction channel for satellite distribution systems," ETSI EN 301-790, December 2000.

${ }^{2}$ DVB, "Satellite component of UMTS, S-UMTS;," ETSI TS 102 744-2-1 V0.0.9, October 2012.

${ }^{3}$ G. Gallinaro and R. Rinaldo, "Assessment of potentiality of adjacent channel interference mitigation in a low-rate TDMA system," in 10th International Workshop on Signal Processing for Space Communications (SPSC 2008), Oct 2008.

${ }^{4}$ M. Moher, W. Zhang, P. Febvre, and J. Rivera-Castro, "Multi-user detection for Inmarsat's BGAN system," in 5th Advanced satellite multimedia systems conference and signal processing for space communications workshop ASMS, Sept. 2010, pp. $135-140$.

${ }^{5}$ NICOLE, "Next generation interactive broadcast mobile," ESA Contract 23030/10/NL/CLP, July 2011.

${ }^{6}$ M. Casadei, S. Cioni, and G. E. Corazza, "Advanced iterative symbol timing recovery for mobile DVB-RCS," in Proceedings of the 65th IEEE Vehicular Technology Conference, VTC Spring 2007, April 2007, pp. 1425 -1429.

${ }^{7}$ M. Flohberger, W. Gappmair, and S. Cioni, "Two iterative algorithms for blind symbol timing estimation of M-PSK signals," in Proceedings of the International Workshop on Satellite and Space Communications, IWSSC 2009, Sept. 2009, pp. $8-12$.

${ }^{8} \mathrm{M}$. Morelli and A. A D'Amico, "Maximum likelihood timing and carrier synchronization in burst-mode satellite transmissions," EURASIP Journal on Wireless Communications and Networking, June 2007, open access : http://jwcn.eurasipjournals.com/content/2007/1/065058.

${ }^{9} \mathrm{M}$. Oerder and H. Meyr, "Digital filter and square timing recovery," IEEE Trans. Commun., vol. 36, no. 5, pp. 605-612, may 1988.

${ }^{10}$ M. Diaz, N. Courville, C. Mosquera, G. Liva, and G. E. Corazza, "Nonlinear interference mitigation for broadband multimedia satellite systems," in Proceedings of the International Workshop on Satellite and Space Communications, IWSSC 2007, Sept. 2007, pp. $61-6$.

${ }^{11}$ G. E. Corazza, P. Salmi, A. Vanelli-Coralli, and M. Villanti, "Differential and non-coherent post detection integration techniques for the return link of satellite W-CDMA systems," in Proceedings of the 13th IEEE International Symposium on Personal, Indoor and Mobile Radio Communications PIMRC 2002, Sept. 2002, pp. 300 - 304. 


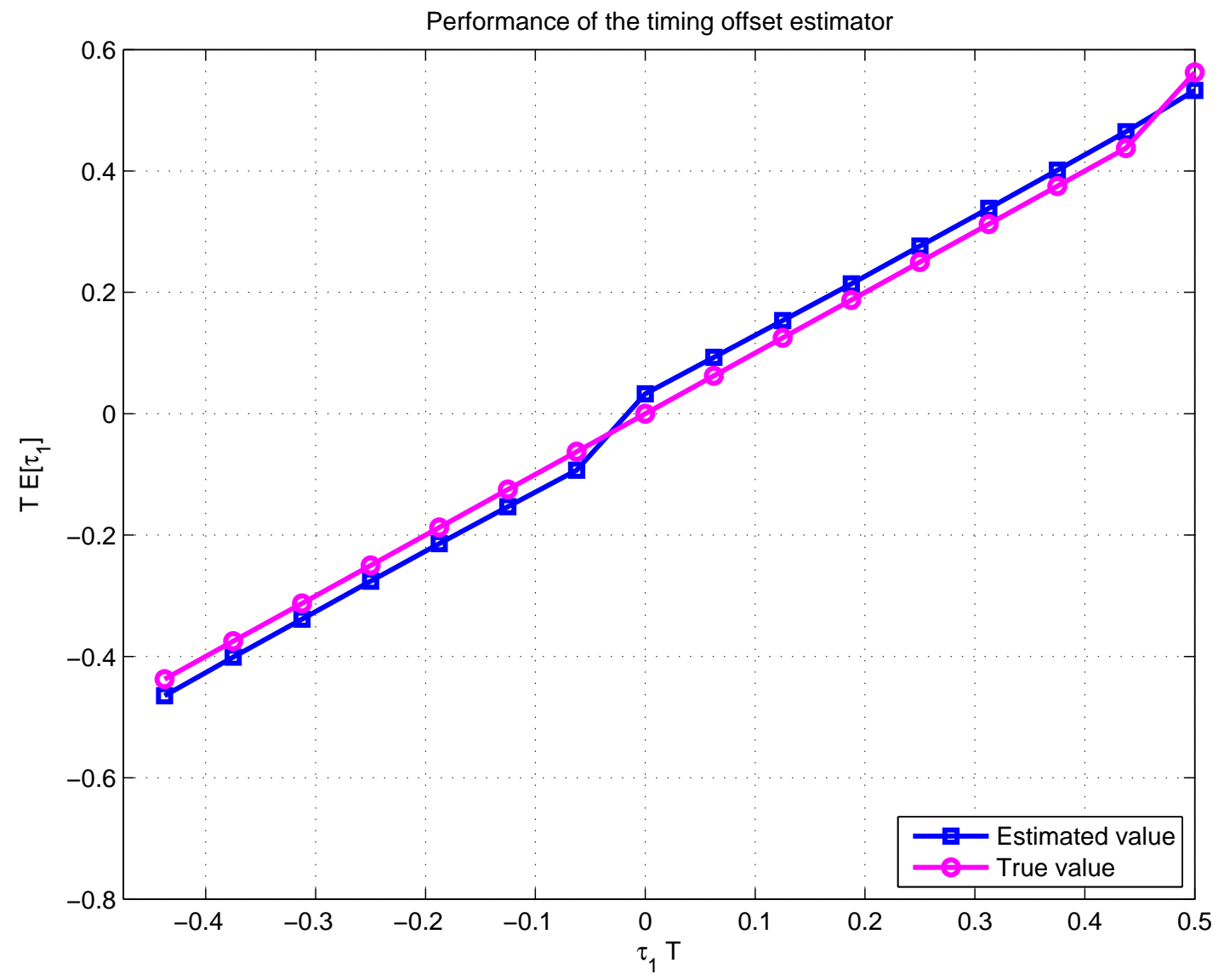

Figure 9. Performance of the timing estimator at $E_{s} / N_{0}$ of $0 d B$

10 of 10 